\title{
Thermal hysteresis of ferromagnetic/antiferromagnetic compensated bilayers
}

\author{
F. I. F. Nascimento, ${ }^{1}$ Ana L. Dantas, ${ }^{1}$ L. L. Oliveira, ${ }^{1}$ V. D. Mello, ${ }^{1}$ R. E. Camley,${ }^{2}$ and A. S. Carriço ${ }^{3, *}$ \\ ${ }^{1}$ Departamento de Física, Universidade do Estado do Rio Grande do Norte, 59610-210 Mossoró, RN, Brazil \\ ${ }^{2}$ Department of Physics, University of Colorado at Colorado Springs, Colorado Springs, Colorado 80918, USA \\ ${ }^{3}$ Departamento de Física Teórica e Experimental, Universidade Federal do Rio Grande do Norte, Campus Universitário, \\ 59078-970 Natal, RN, Brazil
}

(Received 27 June 2009; published 12 October 2009)

\begin{abstract}
We report a theoretical investigation of thermal hysteresis of fourfold anisotropy ferromagnetic (FM) film exchange coupled to a compensated antiferromagnetic substrate. Thermal hysteresis occurs if the temperature interval includes the reorientation transition temperature, below which the frustration of the interface exchange coupling leads to a $90^{\circ}$ rotation of the magnetization of the ferromagnetic layer. The temperature width of the thermal hysteresis is tunable by external magnetic fields of modest magnitude, with values of $43 \mathrm{~K}$ for an external field of $110 \mathrm{Oe}$ and of $14 \mathrm{~K}$ for a field of $210 \mathrm{Oe}$, for a $\mathrm{Fe}(12 \mathrm{~nm}) / \mathrm{MnF}_{2}(110)$ bilayer. For a $\mathrm{Fe}(3 \mathrm{~nm}) / \mathrm{FeF}_{2}(110)$ bilayer the width of the thermal hysteresis is $23 \mathrm{~K}$ at $110 \mathrm{Oe}$ and $13 \mathrm{~K}$ at $300 \mathrm{Oe}$. We discuss how the thickness of the iron film affects the field tuning of the thermal hysteresis width, and also how the thermal loops may be used to identify the nature of the interface exchange energy.
\end{abstract}

DOI: $10.1103 /$ PhysRevB.80.144407

PACS number(s): 75.70.Cn, 75.75.+a

\section{INTRODUCTION}

Thermal hysteresis has recently been investigated in a number of nanostructured magnetic systems, including alloys, ${ }^{1}$ multilayers, ${ }^{2-5}$ thin rare-earth films, ${ }^{6,7}$ and nanometric ferromagnetic (FM) particles exchange coupled to antiferromagnetic (AFM) substrates. ${ }^{8}$ In these structures, there is a small energy difference between two metastable states, which leads to thermal bistability.

The thermal hysteresis in $\mathrm{Fe} / \mathrm{Gd}$ and $\mathrm{Co} / \mathrm{Gd}$ multilayers originates in the large differences in the Curie temperatures and saturation magnetic moments of $\mathrm{Fe}$ and $\mathrm{Gd}$ and the magnetic anisotropy of the $\mathrm{Fe}$ layers. ${ }^{2,3}$ In these systems, the $\mathrm{Fe}$ layers are antiferromagnetically coupled to the Gd layers, and there are two states that are stable at the same temperature. At high temperatures, the system is in an Fe-aligned state (AS), with the iron spins along the external field and with the small Gd moments opposite to the external field. Upon cooling, the thermal average value of the Gd moments increases, but the system may remain in the Fe-aligned state even though the net magnetic moment is opposite to the external field for temperatures below the compensation temperature. The reason for this is that the anisotropy in the Fe layers is sufficient to keep the spin structure in place. Upon further cooling, the Fe-aligned state becomes unstable, and the system reverses to the Gd-aligned state, with the Gd moments parallel to the applied field and the Fe moments opposite to the applied field. A similar effect occurs on heating the system from low temperatures. The Gd-aligned state is held stable by the iron anisotropy as the temperature is increased beyond the compensation temperature. ${ }^{2,3}$ Similar features are also seen in other transition-metal/rare-earth metal multilayers with antiferromagnetic interface exchange coupling. ${ }^{4,5}$

The thermal hysteresis of thin Dy films ${ }^{6}$ is due to the combined effect of the strong temperature dependence of the magnetization and the hexagonal anisotropy, and surface effects which produce interesting modifications in the helical phase in confined geometries. It may be exceptionally large, covering the whole temperature interval between the Curie and the Néel temperatures, and can be strongly tuned by an external field, even though the external field strength (from $0.05 \mathrm{kOe}$ to $1.5 \mathrm{kOe}$ ) is much smaller than the typical values of external field required to induce phase transitions in bulk Dy. Above a critical film thickness, both the width and the efficiency of the field tuning of the thermal hysteresis are large. Both features are due to the nucleation of an alternating helicity state, comprising helices with opposite chirality within the film, and leading to an increased moment in the external field direction, compared with that of a simple helical state. The alternating helicity $(\mathrm{AH})$ state and helical $(\mathrm{H})$ state are both stable at the same temperature. As a result, there are large differences between the isofield magnetization curves, corresponding to heating (AH state) and cooling ( $\mathrm{H}$ state) processes.

Trilayers composed of a Dy film sandwiched between two films of Fe or Gd have additional features, since by adding ferromagnetic films on the outside, one can further modify the effects of an external field on the Dy film. ${ }^{7}$ New magnetic states are formed at temperatures close to the Néel temperature of Dy due to the large Zeeman energy of the ferromagnetic layers. Furthermore, $\mathrm{Fe}$ is antiferromagnetically coupled to Dy at the interfaces while Gd is ferromagnetically coupled to the Dy at the interfaces. This difference in the nature of the interface exchange energy allows substantial changes in how the external field interacts with the structure as a whole, and affects the field tuning of the temperature width of the thermal hysteresis.

We have recently shown ${ }^{8}$ that owing to the temperature change of the interface field, iron dots on an uncompensated antiferromagnetic surface may exhibit room-temperature thermal hysteresis, which is tunable by external fields of moderate strengths (a few kOe). We considered a temperature interval bounded by the Néel temperature of the substrate, and we calculated the heating and cooling curves in the presence of an external field oriented opposite to the interface exchange field. Starting the heating process with 
the dot aligned along the interface field, there is a switch to a field-aligned state near the Néel temperature of the substrate. Upon cooling from this point the field-aligned state is stabilized by the dot's anisotropy until past the value of temperature, where the interface field is larger than the external field. One key point of this system is that the thermal hysteresis may occur well below the critical temperature of the dot. Furthermore, the width of the hysteresis loops depends on the dimensions of the ferromagnetic dot, and can be significant for dots elongated in the direction of the interface field. In this case the shape anisotropy barrier is large and holds the field-aligned state stable up to very small temperature values in the cooling branch.

There is an interesting early report on magnetic phase transitions on compensated ferromagnetic/antiferromagnetic bilayers, involving the switching of the direction for the easy axis of the ferromagnetic layer. ${ }^{9}$ In $\mathrm{Fe} / \mathrm{FeF}_{2}(110)$ bilayers, the $\mathrm{Fe}$ easy axis was found to switch from the [001] direction at $300 \mathrm{~K}$, to the $[1 \overline{1} 0]$ direction at $10 \mathrm{~K}$, when the $\mathrm{FeF}_{2}$ substrate is ordered. The $\pi / 2$ rotation of the Fe spins contributes to lowering the interface exchange energy with the $\mathrm{FeF}_{2}$ spins near the interface slightly canted.

In this paper we present a theoretical investigation of the thermal hysteresis of compensated bilayers consisting of a thin iron film, with an in-plane fourfold anisotropy, exchange coupled to a compensated antiferromagnetic substrate. We consider iron films with thicknesses ranging from 3 to 12 $\mathrm{nm}$, exchange coupled to $\mathrm{MnF}_{2}(110)$ and $\mathrm{FeF}_{2}(110)$ compensated AFM substrates. The point of choosing $\mathrm{MnF}_{2}(110)$ and $\mathrm{FeF}_{2}(110)$ substrates is that these are well-known twosublattices antiferromagnetic materials, often used as model systems for the investigation of new magnetic phenomena of thin films and artificial multilayers. ${ }^{10,11}$ Furthermore, these antiferromagnetic materials have intrinsic exchange fields of similar magnitudes, but have large differences in the anisotropy fields. The anisotropy field of $\mathrm{FeF}_{2}$ is larger than that of $\mathrm{MnF}_{2}$ by over one order of magnitude. As a result $\mathrm{MnF}_{2}$ is much softer, in the sense that variations in angular orientation are allowed over several AF planes. We show below that this has a relevant impact in the field tuning of the thermal hysteresis.

The ferromagnetic spins have two perpendicular directions stabilized by an anisotropy barrier, which is proportional to the thickness of the iron layer. We assume that one of the in-plane easy directions of the iron layer is parallel to the uniaxial axis of the antiferromagnetic substrate. For $\mathrm{MnF}_{2}(110)$ and $\mathrm{FeF}_{2}(110)$ substrates this means that the [001] direction is also an easy axis for the iron layer. We consider a temperature interval bounded by the Néel temperature of the substrate, and we calculate the heating and cooling curves in the presence of an external field $H$ parallel to the in-plane [001] direction.

There are two states with unique properties. One such state is the interface-reoriented state (IRS), shown in Fig. 1(a), in which the FM spins are perpendicular to the external field and the AFM spins are in a transverse canted orientation, slightly off the [001] direction. In the absence of external fields, the IRS forms at low temperature for any value of the interface exchange field. ${ }^{12,13}$ We note that the IRS has the ferromagnetic spins along an easy axis, the [1힐 direction,

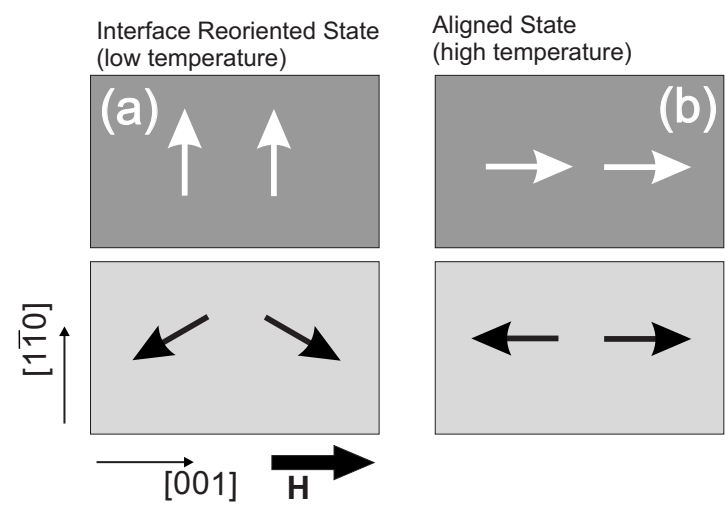

FIG. 1. (a) Schematic representation of the reoriented state, with the iron spins along the $y$ easy axis and the interface spins of the AFM substrate in a transverse canted state; (b) schematic representation of the field-aligned state, with the spins of the iron film along the external field direction and the spins of the interface plane of the AFM substrate in the AFM state.

and has extra stability due to the interface exchange energy. Small values of the external field $H$ leads to small changes in the magnetic structure of the IRS, with the ferromagnetic

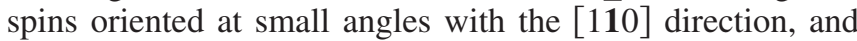
the interface plane antiferromagnetic spins not symmetrically oriented with respect to the [001] direction.

The aligned state shown in Fig. 1(b) has the iron spins along the external field direction, or at small angles with the external field, and the substrate spins along the AFM easy axis. The magnetic moment per unit cell in the AFM interface plane is zero and the net exchange coupling between the FM layer and the substrate is zero. Thus the AS does not benefit from the energy lowering due to interface exchange. However, the Zeeman energy of the iron layer and the intrinsic magnetic energies (exchange and anisotropy energies) of both materials have the minimum values. Near the Néel temperature one may find small deviations from the basic pattern shown in Fig. 1(b), due to spin flips of one of the AFM sublattices in the near-interface region. For the pictures in Fig. 1 we have assumed an antiferromagnetic interaction between the FM layer and the substrate. However, the phenomenology discussed below is equally valid if the interface coupling is ferromagnetic.

The main features of the thermal hysteresis of compensated bilayers may be anticipated from simple arguments. The Fe layer has two perpendicular directions, the [001] and the $[1 \overline{1} 0]$ directions, favored by the anisotropy energy. Thus, there is an anisotropy barrier to be overcome in order to switch from the IRS to the AS and vice-versa. We consider starting at low temperatures and heating the system. In the absence of an external magnetic field, the IRS remains stable throughout the heating curve, because the [1 $\overline{10}$ ] direction is an easy axis of the FM layer. Furthermore, the IRS minimizes the interface exchange energy. ${ }^{12,13}$ Thus even though the interface frustration vanishes at the Néel temperature, or a little after it, when the thermal values of the AFM spins vanish, there is no reason to have a transition. The cooling curve starts in the IRS and so remains until low temperatures. Thus in the absence of external field, the sequence of states along the heating and cooling curves are identical, and there is no thermal hysteresis. 
However, if an external field is applied along the [001] direction it favors a transition from the IRS to the AS. In the heating curve there is a transition driven by the Zeeman energy of the ferromagnetic layer to the aligned state at temperature $T_{1}$. The transition temperature $T_{1}$ depends on the external field strength, the anisotropy of the FM and AFM layers, and the thickness of the FM layer. In the AS the magnetization of the FM layer is along the external field direction ([001], which we shall call the $z$ axis) and the AFM interface spins are in a configuration, which depends on the nature of the interface coupling (either ferromagnetic coupling or antiferromagnetic coupling). In this case the cooling curve starts in the AS and upon cooling one reaches a value of temperature $T_{2}$, with $T_{2}<T_{1}$, where the interface frustration is strong enough to overcome the ferromagnetic anisotropy energy barrier and the Zeeman energy, and the system switches back to the IRS. The width of the thermal hysteresis $\left(\Delta T=T_{1}-T_{2}\right)$ may thus be tuned by the external field strength, the anisotropy energy density and the thickness of the FM layer, the strength of the interface field, and the magnetic properties (exchange and anisotropy energies) of the substrate.

Another interesting feature is that there is a minimum value of the external field strength to make the IRS unstable along the heating curve. For an external field above this critical value there is thermal hysteresis. This threshold value of the external field, $H^{*}$, may be estimated starting with the field strength corresponding to the fourfold anisotropy field of the FM layer. This would be the value of the external field to switch the FM layer from the [1 10$]$ to the [001] direction in the absence of interface exchange energy. For iron the value of the threshold field is $H^{*}=107.6$ Oe. ${ }^{14}$ The interface exchange energy coupling adds stability to the IRS and the actual field required to switch the IRS is larger than $H^{*}$, even though the transition occurs close to the Néel temperature. Therefore, the threshold value of the external field required to produce an IRS-AS switch in the heating branch may depend on whether the interface coupling is antiferromagnetic or ferromagnetic. If the FM layer is ultrathin the magnetic energy may depend on the fine detail of the arrangement of spins in the near-interface region. Thus, as shown in detail below, one may use the thermal hysteresis of compensated bilayers to learn about the nature, ferromagnetic or antiferromagnetic, of the interface exchange energy.

In our model system we consider a bilayer with full compensation at a microscopic length scale, in which the magnetic structure takes no account of possible relaxation effects, either from geometrical or other reasons. The magnetic interactions between the FM layer and the AFM substrate is represented by an exchange-energy term, coupling interface spins from the two materials. Both materials are assumed to have the bulk structure, the AFM interface plane is made of equal number of spins of opposite sublattices and the magnetic interface structure is assumed uniform. The model is applied to $\mathrm{Fe} / \mathrm{FeF}_{2}(110)$ and $\mathrm{Fe} / \mathrm{MnF}_{2}(110)$ systems. It applies as well to bilayers with other intrinsically compensated substrates such as $\mathrm{NiO}(001)$ or $\mathrm{NiO}(110), \mathrm{CoO}(001)$ or $\mathrm{CoO}(110)$, etc. Furthermore, it also applies to bilayers made of uncompensated antiferromagnetic substrates, with average compensation of the interface spin pattern produced by interface roughness.
We note that there have been a number of theoretical studies of interface phase transitions in FM/AFM structures, however, none of these examined thermal hysteresis. Some of these papers will be discussed in Sec. II, but we wish to point out two relevant papers at this point. A variety of bilayers and other structures have been studied, ${ }^{15}$ with an emphasis on simple analytic expressions in special cases. The effect of the external magnetic field, a critical issue in this paper, was neglected. An interesting study focusing on spinflop transitions in the antiferromagnet produced by very large magnetic fields has been reported. ${ }^{16}$ This work neglected the anisotropy in the ferromagnet, but did indicate a different method to evaluate the sign of the coupling between the ferromagnet and antiferromagnet.

\section{MODEL}

We study compensated bilayers with the stacking pattern of the $\mathrm{Fe} / \mathrm{MnF}_{2}(110)$ and $\mathrm{Fe} / \mathrm{FeF}_{2}(110)$ systems. The AFM substrate is a stacking of AFM planes containing moments from the two sublattices. Spins from a given sublattice in the same plane are considered equivalent, and to each plane we assign two spin variables. Except for the surface spins, the coordination number is $z=8$. Each AFM spin has four nearest neighbors (NNs) (of the opposite sublattice) in the same plane, and two nearest neighbors in the two neighboring planes. The normal to the surface is in the $y$-axis direction, the easy directions of the in-plane fourfold anisotropy of the FM material are along the $x$ axis and the $z$ axis, and the uniaxial anisotropy of AFM is along the $z$ axis. The magnetic structure of the substrate is represented by the angles that each spin makes with the $z$ axis $\left[\theta_{n}(1), \theta_{n}(2), n=1, \ldots N_{\mathrm{AFM}}\right]$, and the thermal average value of the spins $\left[\left\langle S_{n}(1)\right\rangle,\left\langle S_{n}(2)\right\rangle, n=1, \ldots, N_{\mathrm{AFM}}\right] \theta_{n}(1)$ and $\theta_{n}(2)$ are the angles with respect to the easy axis for spins of sublattices 1 and 2 at the $n$th plane and $N_{\mathrm{AFM}}$ is the number of (110) planes in the AFM film. The ferromagnetic film with $N_{\mathrm{FM}}$ planes is represented in a similar manner, and the bilayer magnetic structure is described in terms of two interacting linear chains, each of which with $\left(N_{\mathrm{AFM}}+N_{\mathrm{FM}}\right)$ spins.

The magnetic energy is given by

$$
\begin{aligned}
E= & \sum_{n=\mathrm{FM}} K_{1} S_{z, n}^{2} S_{x, n}^{2}-\sum_{n=\mathrm{AFM}} K S_{z, n}^{2} \\
& -\sum_{n} g \mu_{B} \vec{S}_{n} \cdot \vec{H}-\sum_{n m} J_{n m} \vec{S}_{n} \cdot \vec{S}_{m},
\end{aligned}
$$

where the first term is the fourfold anisotropy of Fe, the second term is the uniaxial anisotropy of AFM, the third term is the Zeeman energy, and the last term is the exchange energy. For the Fe spins we use $S=1, g=2$, and fourfold anisotropy constant $K_{1}=3.5 \times 10^{4} \mathrm{~J} / \mathrm{m}^{3}$, which amounts to an anisotropy field of $H_{a}=2 K_{1} / M_{S}=0.4 \mathrm{kOe}$, with $M_{S}=1.7 \times 10^{6} \mathrm{~A} / \mathrm{m}$, and a distance between neighboring (110) atomic planes of $1.5 \AA$. Because of the strong exchange coupling in $\mathrm{Fe}$, the $\mathrm{Fe}$ spins are effectively rigidly coupled to each other. These parameters reproduce the resonance frequency of thin Fe films. ${ }^{17}$

For the antiferromagnetic materials we have selected the values of the magnetic parameters so as to reproduce the 
Néel temperatures and the ratios between the magnitude of the exchange and anisotropy fields. ${ }^{18,19}$ For $\mathrm{FeF}_{2}$ we have used $S=1, g=2$, anisotropy field $\left(H_{a}=2 K / g \mu_{B} S\right)$ of 149 kOe, exchange field $\left(H_{e}=8 J S / g \mu_{B}\right)$ equal to $434 \mathrm{kOe}$, and Néel temperature $T_{N}=78 \mathrm{~K}$. For $\mathrm{MnF}_{2}$ we have chosen $S=2.5, g=2, H_{a}=8.8 \mathrm{kOe}, H_{e}=540 \mathrm{kOe}$, and $T_{N}=67 \mathrm{~K}$. The exchange energy coupling the spins across the FM/AFM interface was assumed to be equal to the exchange energy of the AFM substrate.

The equilibrium pattern is found by requiring that each magnetic moment along the chains be parallel to the local effective field. Thermal effects are taken into account using a local mean-field approach, where the thermal average magnetic moment of each spin, $\langle\vec{m}\rangle=g \mu_{B}\langle\vec{S}\rangle$, is calculated using $\langle\vec{m}\rangle=g \mu_{B} S B_{S}\left(\frac{g \mu_{B} S\left\langle\vec{H}_{e f f}\right\rangle}{k_{B} T}\right)$, where $B_{S}\left(\frac{g \mu_{B} S\left\langle\vec{H}_{e f f}\right\rangle}{k_{B} T}\right)$ is the Brillouin function, $\langle\cdots\rangle$ denotes a thermal average, and the effective field $\vec{H}_{e f f}$ is obtained from the spin gradient of the magnetic energy. The spin orientation in the planes and the thermal values of the spins are both calculated self-consistently, using the method developed earlier and applied to the study of magnetic multilayers and AFM films. ${ }^{18-20}$

We point out that the calculational method used here assumes temperature-independent anisotropy constants, with the temperature dependence of the anisotropy energy coming through the mean-field treatment of the spin magnitudes. In fact, the temperature dependence of the anisotropy may be somewhat faster in reality than is indicated by this method. ${ }^{21,22}$ This should not be a problem for the ferromagnet, because the temperatures of interest here are all below $100 \mathrm{~K}$, while the Curie temperature is above 1,000 K. Thus the expected changes in anisotropy in Fe are relatively small, in agreement with recent experiments. ${ }^{23}$ Even if the anisotropy fields in the antiferromagnet decrease more quickly than expected from the mean-field treatment the general features of the behavior should not change substantially. As we will see, the evidence for this is seen in the fact that the $\mathrm{Fe} / \mathrm{FeF}_{2}$ and the $\mathrm{Fe} / \mathrm{MnF}_{2}$ structures have quite similar results for the width of the thermal hysteresis even though the anisotropies differ by a factor of about 15 .

We start the calculation in the heating branch at low temperatures, where a magnetic configuration close to the IRS is expected. Thus, for any value of the external field, we initialize the variables at the IRS. This leads to a rapid convergence of the numeric procedure, in particular, for small values of the external field. For each subsequent value of the temperature, throughout both branches of the thermal loop, the self-consistent procedure is initialized with the magnetic state corresponding to the equilibrium state of the previous value of the temperature. Proceeding this way we find the metastable equilibrium state nearest to the preceding one, as appropriate to modeling the thermal hysteresis phenomena.

We focus on the effects of varying the external field strength and the thickness of the FM layer. The number of AFM planes was chosen so as to allow a full relaxation of the AFM magnetic structure within the AFM layer, starting with the transverse canted magnetic pattern at the interface AFM plane, down to the AFM order, with the AFM spins along the easy axis, at the free surface of the AFM layer. For both materials $\left(\mathrm{MnF}_{2}\right.$ and $\left.\mathrm{FeF}_{2}\right) N_{\mathrm{AFM}}=20$ was enough to

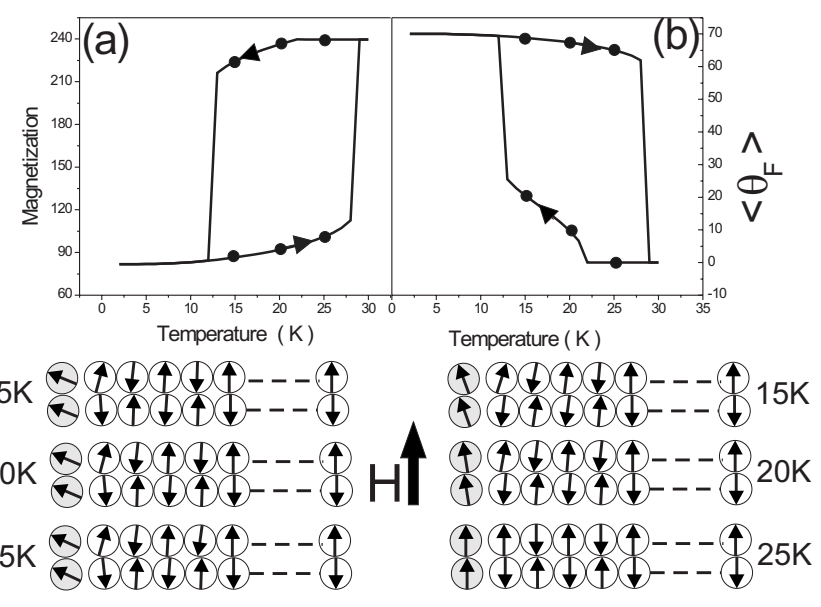

FIG. 2. (a) Component of magnetization along $z$ axis for a $\mathrm{Fe}(9 \mathrm{~nm}) / \mathrm{MnF}_{2}(110)$ bilayer, in units of $\mu_{\mathrm{B}}$ and (b) average angle of FM spins with the [001] direction for a thermal loop under an external field $H=190$ Oe along the $z$ axis. In the spin panels on the left we show schematically the configuration of spins of FM layer (gray shaded symbols) and the AFM substrate for selected points in the heating branch. The panels on the right are for the cooling branch at the same values of temperature.

fulfill this condition throughout the thermal loops, for the chosen values of external field strength and the FM layer thickness.

The model used in this calculation corresponds to energy minimization. Such a scheme was used, for instance, by $\mathrm{Koon}^{24}$ in finding the magnetic hysteresis curves for the FM/ AFM structure. However, it was later shown that this model was inadequate for these calculations because it did not take into account an instability that occurs in dynamic calculations, which focus on the true time evolution of the structure. ${ }^{25,26}$ In the present case, however, where we calculate the thermal hysteresis curve, the instability should not occur because the magnetic structure in the antiferromagnet is always close to its ground state and does not have the large deviations seen in the twisted states found in a magnetic hysteresis experiment. ${ }^{27}$

\section{RESULTS AND DISCUSSIONS}

In Fig. 2 we show a typical thermal loop for a $\mathrm{Fe}(9 \mathrm{~nm}) / \mathrm{MnF}_{2}(110)$ bilayer with an external field of $H=190$ Oe along the $z$ axis. Starting at $T=2 \mathrm{~K}$ with the bilayer in the IRS, the transition to the AS phase occurs at $T=29 \mathrm{~K}$ with the FM spins making an average angle of $\left\langle\theta_{F}\right\rangle \approx 62^{\circ}$ with the $z$ axis. Upon cooling from $T=30 \mathrm{~K}$ there is a gradual switch back to the IRS starting at $21 \mathrm{~K}$, closing the thermal hysteresis loop at $T=12 \mathrm{~K}$, corresponding to a temperature width of $17 \mathrm{~K}$. The deviations of the spins in the AFM interface plane from the AFM easy axis in both branches of the thermal loop are smaller than an angle of $15^{\circ}$ and the relaxation of the interface magnetic pattern involves at most five AFM atomic planes throughout the thermal loop.

Looking at the results shown in Fig. 3 for the $\mathrm{Fe}(12 \mathrm{~nm}) / \mathrm{MnF}_{2}(110)$ bilayer, one finds that there is a large decrease in the width of the thermal hysteresis with the 


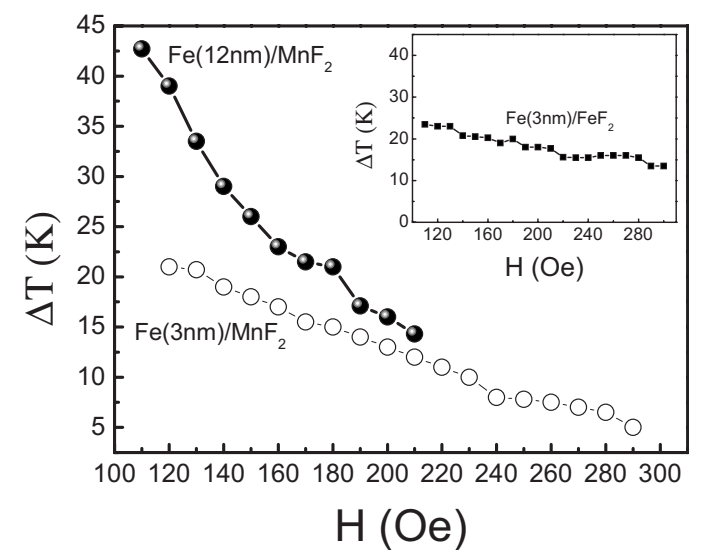

FIG. 3. Width of the thermal hysteresis for $\mathrm{Fe}(12 \mathrm{~nm}) / \mathrm{MnF}_{2}(110)$ and $\mathrm{Fe}(3 \mathrm{~nm}) / \mathrm{MnF}_{2}(110)$ bilayers, and for a $\mathrm{Fe}(3 \mathrm{~nm}) / \mathrm{FeF}_{2}(110)$ bilayer (in the inset).

strength of the external field, starting at $\Delta T=43 \mathrm{~K}$ for $H=110 \mathrm{Oe}$, and going down to $\Delta T=14 \mathrm{~K}$ for $H=210$ Oe. This corresponds to closed thermal loops, similar to that shown in Fig. 2. The field dependence of $\Delta T$ originates in the fact that both the temperatures $T_{1}$ (for the IRS-AS transition) and $T_{2}$ (for the AS-IRS transition) decrease with the magnitude of $H$, however $T_{1}$ decreases faster. For instance: for $H=110 \mathrm{Oe}, T_{1}=62 \mathrm{~K}$ and $T_{2}=20 \mathrm{~K}$; for $H=150 \mathrm{Oe}$, $T_{1}=43 \mathrm{~K}$ and $T_{2}=16 \mathrm{~K}$; for $H=190$ Oe, $T_{1}=29 \mathrm{~K}$ and $T_{2}=12 \mathrm{~K}$; and for $H=200 \mathrm{Oe}, T_{1}=26 \mathrm{~K}$ and $T_{2}=10 \mathrm{Oe}$.

The decrease in the temperature width of the thermal hysteresis with the magnitude of the external field can be understood using a simple argument. If the AFM interface spins were not stabilized by the FM layer then at the Néel temperature there would be no interface effect, the extra stability of the IRS coming from the interface energy would vanish, and a field of $H=H^{*}$ (the FM anisotropy field) would produce a IRS-AS transition. By increasing the value of the external field strength beyond $H^{*}$ one should be able to overcome the total-energy barrier, which holds the IRS stable at a value of temperature smaller than $T_{N}$. The larger the value of the external field strength the lower is the value of $T_{1}$. This is the reason for the field dependence of $T_{1}$, except for the fact that there is an interface stabilization of the AFM interface spins and the minimum value of the external magnetic field to produce the IRS-AS switch is larger than $H^{*}$. The temperature $T_{2}$, corresponding to the lower limit of the thermal hysteresis, is associated with the instability of the AS in the cooling branch. The energy barrier holding the AS in place comes from the anisotropy of the FM layer and the Zeeman energy. From the point of view of the Zeeman energy one might think that when the external field is made larger it is possible to hold the AS stable until lower temperatures. On the other hand the interface exchange energy is smaller in the IRS. The transition from the AS to the IRS at low temperatures comes as a result of the balance between the anisotropy energy, Zeeman energy, and the interface exchange energy. The weaker dependence of $T_{2}$ on the value of the external field strength is due to the fact that at low temperatures the AFM spins have thermal average values near those of saturation, and the field effect on the interface energy is smaller.

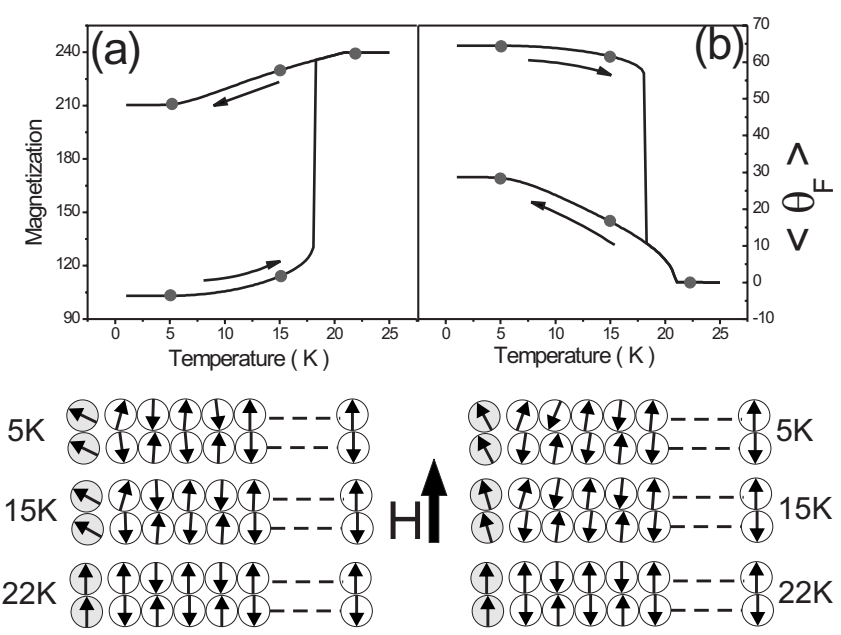

FIG. 4. (a) Component of magnetization along $z$ axis for a $\mathrm{Fe}(9 \mathrm{~nm}) / \mathrm{MnF}_{2}(110)$ bilayer, in units of $\mu_{\mathrm{B}}$ and (b) average angle of FM spins with the [001] direction for a thermal loop under an external field $H=220$ Oe along the $z$ axis. In the panels on the left we show schematically the configuration of spins of FM layer (gray shaded symbols) and the AFM substrate for selected points in the heating branch. The panels on the right are for the cooling branch at the same values of temperature.

Thus by changing the external field one gets small changes in the value of the temperature $T_{2}$, for the AS-IRS transition.

The results shown in Fig. 3 for the thinner ferromagnetic layers, the $\mathrm{Fe}(3 \mathrm{~nm}) / \mathrm{MnF}_{2}$ and the $\mathrm{Fe}(3 \mathrm{~nm}) / \mathrm{FeF}_{2}$ structures, indicate that the field effect on the width of the thermal hysteresis is much weaker for the thin Fe films. In both cases the interface energy is a larger fraction of the total energy of the bilayer. Therefore the changes produced by the external field in the values of the transition temperatures $T_{1}$ and $T_{2}$, are smaller than for the $\mathrm{Fe}(12 \mathrm{~nm}) / \mathrm{MnF}_{2}(110)$ bilayer. Also, field tuning of the thermal hysteresis is stronger in $\mathrm{Fe}(3 \mathrm{~nm}) / \mathrm{MnF}_{2}$ than in $\mathrm{Fe}(3 \mathrm{~nm}) / \mathrm{FeF}_{2}$, because for the later case there is a much larger contribution of the AFM anisotropy to the magnetic energy of the bilayer. Thus, the Zeeman energy is a smaller fraction of the total magnetic energy.

The IRS is stabilized by the interface exchange energy, which is proportional to the thermal average value of the antiferromagnetic spins at the interface. Since the interface exchange energy becomes small at sufficiently high temperature, the IRS-AS transition in the heating branch is certain to occur if the external field is strong enough to overcome the anisotropy barrier of the ferromagnetic layer. The same is not true for the AS-IRS transition. By increasing the external field one may reach a value of $H$ for which the AS-IRS transition in the cooling branch does not occur. In this case starting at low temperatures in the IRS, there is a transition to the AS at some temperature $T_{1}$, and upon cooling the bilayer back to the initial value of temperature the system does not return to the original IRS. In Fig. 4 we show an example of this case, for a $\mathrm{Fe}(9 \mathrm{~nm}) / \mathrm{MnF}_{2}(110)$ bilayer, subjected to an external field of 220 Oe. As shown in Fig. 4, starting at low temperatures in the IRS, with the FM spins making an average angle of $\left\langle\theta_{F}\right\rangle \approx 64^{\circ}$ with the $z$ axis, and a low value of the magnetization along the external field direc- 
tion, there is a transition to the AS around $T=18 \mathrm{~K}$, and by heating a little further, one reaches saturation of the magnetization in the external field direction around $T=21 \mathrm{~K}$. The Zeeman energy plays a dominant role in the energy balance and the sequence of magnetic states along the cooling branch. Starting at $T=25 \mathrm{~K}$ with the bilayer in the AS, and the magnetization saturated along the external field, the cooling branch is a sequence of AS, all the way down to very low temperatures, with the ferromagnetic spins at small angles with the external field. The average angle of the $\mathrm{Fe}$ spins with the $z$ axis evolves from $\left\langle\theta_{F}\right\rangle=0$ at $T=21 \mathrm{~K}$ and, by completing the thermal loop, reaches the value of $\left\langle\theta_{F}\right\rangle \approx 28^{\circ}$ at $T=5 \mathrm{~K}$. Notice that the ferromagnetic anisotropy energy is nearly the same in the initial and final states of the thermal loop. Starting in the IRS at $T=5 \mathrm{~K}$ in the heating branch with $\left\langle\theta_{F}\right\rangle \approx 64^{\circ}$, the thermal loop ends with the system in the AS with $\left\langle\theta_{F}\right\rangle \approx 28^{\circ}$ at $T=5 \mathrm{~K}$ in the cooling branch. However, the Zeeman energy is large enough to inhibit the transition to the IRS at low temperatures. We have found that for larger values of the external field strength the high-temperature state stays stable till low temperatures and the thermal loop produces a switch from an initial magnetic state to another final state. This is a point that might be of some interest for magnetoelectronic applications, in which device operation involves heating of the interface between a ferromagnetic thin film and an antiferromagnetic substrate.

The IRS-AS transition in the heating branch of the thermal loop of an $\mathrm{Fe}(9 \mathrm{~nm}) / \mathrm{MnF}_{2}(110)$ bilayer occurs at temperatures of $T_{1}=29 \mathrm{~K}$, for an external field of $190 \mathrm{Oe}$, and at $T_{1}=18 \mathrm{~K}$ for an external field strength of 220 Oe. In both cases $T_{1}$ is well below the Néel temperature of the $\mathrm{MnF}_{2}$ substrate, and the thermal average value of the antiferromagnetic spins is near the saturation value at the transition temperature. For the examples considered up to this point, the energy balance at the transition temperature does not depend strongly on the nature (ferromagnetic or antiferromagnetic) of the interface exchange coupling. We have found (results not shown) that the IRS-AS transition in the heating branch occurs at roughly the same temperatures for either antiferromagnetic or ferromagnetic interface coupling.

By making the $\mathrm{Fe}$ layer thinner and reducing the external field strength, one may upshift the transition temperature $T_{1}$ to very close to the Néel temperature. In this temperature limit the magnetic order in the substrate is limited to a few planes from the interface. The spins in the antiferromagnets near the interface are stabilized by the interface exchange energy with the ferromagnetic layer and the external field. The remaining spin in the antiferromagnet have thermal average moments, which are close to zero. In essence there is a liquid moment for the spins in the antiferromagnet just at the interface when they are small in magnitude and only weakly stabilized.

Most interestingly, the sign of the interface exchange constant can be inferred from the thermal loops of the thin FM layers under the action of an external field smaller than the ferromagnet anisotropy field. In Fig. 5 we show that the thermal loop of an $\mathrm{Fe}(3 \mathrm{~nm}) / \mathrm{MnF}_{2}(110)$ bilayer under an external field $H=104$ Oe exhibits thermal hysteresis if the interface exchange energy is ferromagnetic. We also show in Fig. 5 that there is no thermal hysteresis if the interface exchange energy is antiferromagnetic.

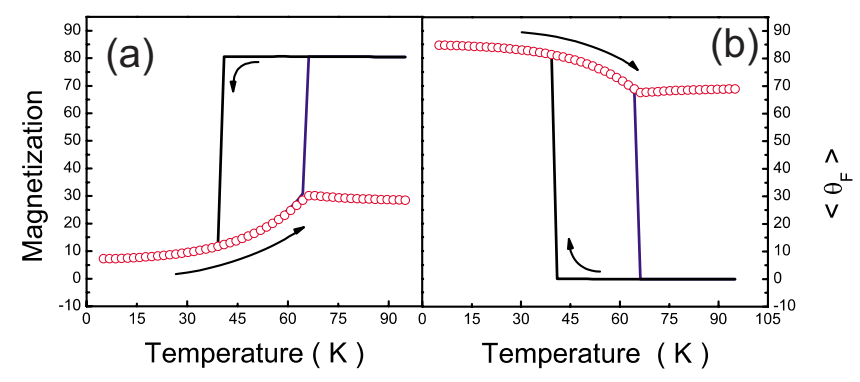

FIG. 5. (Color online) (a) Component of magnetization along $z$ axis in units of $\mu_{\mathrm{B}}$ and (b) average angle of FM spins with the [001] direction for a thermal loop of a $\mathrm{Fe}(3 \mathrm{~nm}) / \mathrm{MnF}_{2}(110)$ bilayer under an external field $H=104$ Oe. The continuous line curves and open symbol curves correspond to FM and AFM interface exchange energies.

The two cases differ in the arrangements of the interface spins in the antiferromagnet at elevated temperatures. For ferromagnetic interface exchange coupling, the net moment of the substrate spins at the interface plane is slightly larger than in the case of antiferromagnetic interface exchange coupling. This rather small difference leads to a relevant difference in the effective exchange field on the ferromagnetic layer as we will see below.

Near the Néel temperature the net moment of the AFM spins at the interface plane, points in a direction very close to the exchange field produced by the ferromagnetic interface spins. For ferromagnetic (antiferromagnetic) interface exchange coupling, the liquid moment of the AFM spins at the interface plane are nearly parallel (antiparallel) to the direction of the FM spins. The magnetic order of the substrate spins is almost entirely due to the exchange field produced by the interface ferromagnetic spins. However, there is also a small contribution from the external field. For ferromagnetic (antiferromagnetic) coupling the spins at the substrate interface plane are thermally stabilized by an effective field with $z$ component on the order of $10^{5} \mathrm{Oe}$, the interface exchange field, plus (minus) the external field of 104 Oe. Thus, for ferromagnetic interface exchange coupling, the thermal average value of the AFM interface spins is slightly larger. As a result, the $z$-axis component of the liquid moment of the AFM spins at the interface plane is slightly larger than for the case of antiferromagnetic interface exchange coupling.

For low temperatures, as shown in Fig. 6, the absolute value of the $z$-axis component of the liquid moment $\left|\left\langle\delta S_{I N T}\right\rangle\right|$ of the AFM interface spins has the same value for either ferromagnetic or antiferromagnetic interface exchange coupling. In both cases $\left|\left\langle\delta \mathrm{S}_{I N T}\right\rangle\right|$ turns larger near the Néel temperature, reaching $\left|\left\langle\delta S_{I N T}\right\rangle\right|=0.29$, for ferromagnetic coupling, and $\left|\left\langle\delta S_{I N T}\right\rangle\right|=0.25$ for antiferromagnetic coupling. The small difference of about 0.04 , amounts to $1.6 \%$ of the saturation value of a $\mathrm{MnF}_{2}$ spin. However, the corresponding difference in the $z$-axis component of the exchange field on the ferromagnetic interface spins is not negligible. As shown in Fig. 6 (right vertical axis), near the Néel temperature it is on the order of $1 \mathrm{kOe}$, favoring the transition to the AS for ferromagnetic interface coupling. This increase in the effective field allows the ferromagnetic moments to cross the hard axis and to be stabilized along the field in the aligned state as seen in Fig. 5. 


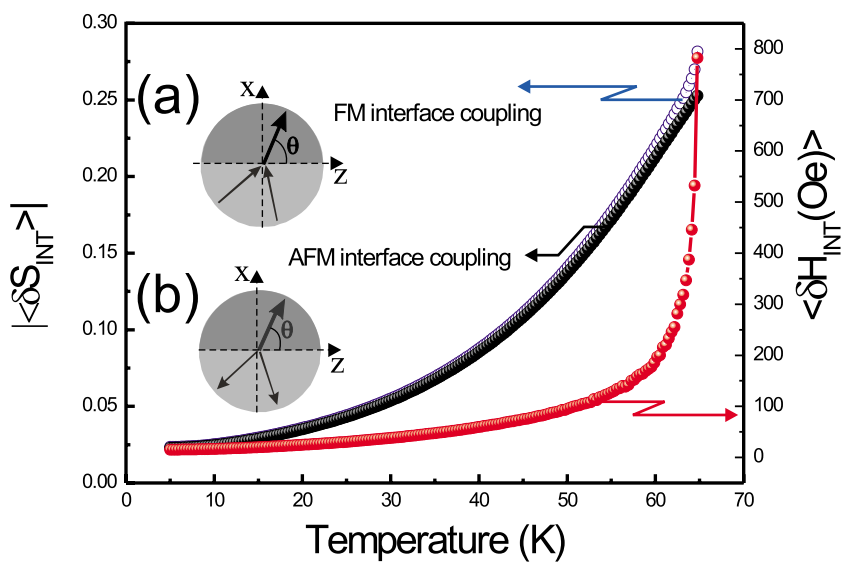

FIG. 6. (Color online) Absolute value of the net moment of the interface AFM spins along the $z$ axis and the exchange field on the FM interface spins along the heating branch of the thermal loop of a $\mathrm{Fe}(3 \mathrm{~nm}) / \mathrm{MnF}_{2}(110)$ bilayer under an external field $H=104$ Oe. The open symbol blue curve and the full symbol black curve correspond to the absolute value of the net moment of the interface spins for FM and AFM interface exchange energies. The open symbol red curve (right vertical axis) shows the difference in the interface exchange field on the FM interface spins between the FM and AFM coupling cases. The insets represent schematically the interface spin configurations for (a) FM and (b) AFM interface coupling.

The above phenomenology relies on rather small differences in the orientation of the interface spins according to the nature of the interface exchange energy. For ferromagnetic interface coupling the transition occurs at a temperature of $66.2 \mathrm{~K}$, corresponding to $98 \%$ of the Néel temperature. We have also examined the case of $\mathrm{FeF}_{2}$ substrate (results not shown). For a $\mathrm{Fe}(3 \mathrm{~nm}) / \mathrm{FeF}_{2}$ bilayer and an external field of 54 Oe there is an IRS-AS transition at $76.99 \mathrm{~K}$ for ferromagnetic interface coupling and no thermal hysteresis in the case of antiferromagnetic interface coupling. Again the transition occurs at approximately $98 \%$ of the Néel temperature, suggesting that one may use thermal hysteresis to investigate the nature of the interface exchange coupling.

We have found that the range of the external field strengths that allows one to determine the kind of exchange interaction at the interface is a narrow field interval just below $H^{*}$. For external field strengths larger than the anisotropy field of the ferromagnet, there is thermal hysteresis for both kinds of coupling. For $H$ much smaller than $H^{*}$, there is no thermal hysteresis for either ferromagnetic or antiferromagnetic coupling. The minimum value of the external field strength required for thermal hysteresis for ferromagnetic coupling is slightly smaller than that required in the case of antiferromagnetic coupling. In the case of the $\mathrm{Fe}(3 \mathrm{~nm}) / \mathrm{MnF}_{2}(110)$ bilayer, for ferromagnetic coupling there is thermal hysteresis for $H$ larger than 104 Oe and for antiferromagnetic coupling the minimum value of external field is 105.5 Oe.

We have also investigated the effect of increasing the FM anisotropy. For a $50 \%$ increase in the anisotropy (from 0.4 $\mathrm{kOe}$, as used in the paper for iron, to $0.6 \mathrm{kOe}$ ) the critical field $H^{*}$ increases from 107.6 Oe (as used in the paper) to
161.4 Oe. In this case we have found that for ferromagnetic coupling there is thermal hysteresis if the external field strength is larger than 158 Oe, whereas for antiferromagnetic coupling the minimum value of external field is 160.5 Oe.

In our present investigation the frustration in the interface exchange coupling is due to the microscopic structure of the stacking of AFM planes, leading to an interface plane with full compensation at a microscopic length scale (equal number of spins of opposite sublattices are present at the interface plane magnetic unit cell). This model applies to intrinsically compensated FM/AFM bilayers, consisting of two layers (the ferromagnetic film and the antiferromagnetic substrate) with ideal magnetic structure, identical to that of the bulk of each material.

The model presented here involves the coupling of a ferromagnet to a perfectly compensated antiferromagnetic surface. Clearly, roughness might change the compensation. This has been investigated theoretically, for example, in Refs. 28 and 29. One feature that came out of these studies is that as the system moves away from the compensated structure, the state illustrated in Fig. 1(a) changes in that the ferromagnet spins cant in the direction of one of the sublattices of the antiferromagnet. In the normal magnetic hysteresis measurement, this leads to a shifting in the hysteresis curve so that the hysteresis loop is no longer centered at zero field. For the thermal hysteresis considered here, it could mean that the thermal hysteresis curves should depend on the direction of the applied field, i.e., whether the field is applied parallel to one sublattice of the antiferromagnet or the other.

The present discussion may also apply to systems with compensation of the interface spin pattern on an average over interface areas of mesoscopic dimensions. Two interesting examples of current interest are FM/AFM bilayers with interface energy compensation due to interface roughness $^{30-32}$ and vicinal bilayers. ${ }^{33}$ Field-tunable thermal hysteresis is likely to occur in these exchange-coupled FM/ AFM bilayers, ${ }^{30-33}$ provided that the interface magnetic structure is compensated at a length scale smaller than the exchange length of the ferromagnetic material.

In summary, we have investigated thermal bistability of thin iron layers, with thicknesses of a few nanometers, exchange coupled to compensated $\mathrm{MnF}_{2}(110)$ and $\mathrm{FeF}_{2}(110)$ substrates. At high temperatures the system is in a fieldaligned state, with the iron layer spins along the external field direction and the net interface exchange energy is zero. At low temperatures, with the thermal average value of the substrate spins near saturation, the frustration of the interface exchange energy leads to a $\pi / 2$ rotation of the iron layer spins to the direction perpendicular to the AFM easy axis and the formation of a liquid magnetic moment in the substrate interface plane oriented parallel (for ferromagnetic interface exchange energy) or antiparallel (for antiferromagnetic interface exchange energy) to the magnetization of the iron layer. The anisotropy barrier separating the high-temperature state, controlled by the Zeeman energy, and the low-temperature state, controlled by the interface exchange coupling, leads to thermal bistability in a temperature interval below the Néel temperature, which is tunable by external field strengths on the order of a few hundred Oe. 
Also, the nature of the interface exchange coupling (either ferromagnetic or antiferromagnetic) may be investigated from the thermal loops of bilayers made of thin iron layers and for small values of the external field strength.

We have also shown that for large values of the external field strength the high-temperature state stays stable throughout the cooling branch of thermal loop down to low temperatures, and the thermal loop produces a switch from an initial magnetic state (IRS) to another final state (AS). We argue that this may have interest for magnetoelectronics applications if device operation involves heating of the interface between a ferromagnetic film and an antiferromagnetic substrate

\section{ACKNOWLEDGMENTS}

The authors acknowledge financial support from CAPES and FAPERN. The work of A.S.C. was supported by CNPq under Grant No. 350773 and the work of A.L.D. was supported by $\mathrm{CNPq}$ under Grant No. 309676. The work of R.E.C. was supported by DOA under Grant No. W911NF-04-1-0247.

\footnotetext{
*Corresponding author. FAX: +55-84-32153791; acarrico@dfte.ufrn.br

${ }^{1}$ S. Demirtas, R. E. Camley, and A. R. Koymen, Appl. Phys. Lett. 87, 202111 (2005).

${ }^{2}$ R. E. Camley, W. Lohstrohb, G. P. Felcherc, N. Hosoitod, and H. Hashizume, J. Magn. Magn. Mater. 286, 65 (2005).

${ }^{3}$ S. Demirtas, M. R. Hossu, R. E. Camley, H. C. Mireles, and A. R. Koymen, Phys. Rev. B 72, 184433 (2005).

${ }^{4}$ M. R. Hossu, Y. Hao, and A. R. Koymen, J. Phys.: Condens. Matter 20, 215224 (2008).

${ }^{5}$ J. P. Andrés, J. A. González, T. P. A. Hase, B. K. Tanner, and J. M. Riveiro, Phys. Rev. B 77, 144407 (2008).

${ }^{6}$ A. L. Dantas, R. E. Camley, and A. S. Carriço, IEEE Trans. Magn. 42, 2942 (2006).

${ }^{7}$ A. L. Dantas, R. E. Camley, and A. S. Carriço, Phys. Rev. B 75, 094436 (2007).

${ }^{8}$ A. L. Dantas, A. S. W. T. Silva, G. O. G. Rebouças, A. S. Carriço, and R. E. Camley, J. Appl. Phys. 102, 123907 (2007).

${ }^{9}$ T. J. Moran, J. Nogués, D. Lederman, and I. K. Schuller, Appl. Phys. Lett. 72, 617 (1998).

${ }^{10}$ J. Nogues, D. Lederman, T. J. Moran, and I. K. Schuller, Phys. Rev. Lett. 76, 4624 (1996).

${ }^{11}$ Z.-P. Li, J. Eisenmenger, C. W. Miller, and I. K. Schuller, Phys. Rev. Lett. 96, 137201 (2006).

${ }^{12}$ M. L. Silva, A. L. Dantas, and A. S. Carriço, J. Magn. Magn. Mater. 292, 453 (2005).

${ }^{13}$ M. L. Silva, A. L. Dantas, and A. S. Carriço, Solid State Commun. 135, 769 (2005).

${ }^{14}$ The value of $H^{*}$ may be calculated from the analysis of stability of the bulk equilibrium state, considering the energy density composed of the fourfold anisotropy plus Zeeman energy.

${ }^{15}$ P. J. Jensen, H. Dreysse, and M. Kiwi, Eur. Phys. J. B 46, 541 (2005).

${ }^{16}$ N. Cramer and R. E. Camley, Phys. Rev. B 63, 060404(R)
}

(2001).

${ }^{17}$ Yu. V. Goryunov, N. N. Garif'yanov, G. G. Khaliullin, I. A. Garifullin, L. R. Tagirov, F. Schreiber, Th. Mühge, and H. Zabel, Phys. Rev. B 52, 13450 (1995).

${ }^{18}$ A. S. Carriço and R. E. Camley, Phys. Rev. B 45, 13117 (1992).

${ }^{19}$ A. S. Carriço and R. E. Camley, Solid State Commun. 82, 161 (1992).

${ }^{20}$ A. L. Dantas and A. S. Carriço, Phys. Rev. B 59, 1223 (1999).

${ }^{21}$ H. B. Callen and E. Callen, J. Phys. Chem. Solids 27, 1271 (1966).

${ }^{22}$ Y. Millev and M. Fahnle, Phys. Rev. B 51, 2937 (1995).

${ }^{23}$ L. Wee, R. L. Stamps, L. Malkinski, and Z. Celinski, Phys. Rev. B 69, 134426 (2004); B. Heinrich, Z. Celinski, J. F. Cochran, A. S. Arrott, and K. Myrtle, J. Appl. Phys. 70, 5769 (1991).

${ }^{24}$ N. C. Koon, Phys. Rev. Lett. 78, 4865 (1997).

${ }^{25}$ T. C. Schulthess and W. H. Butler, Phys. Rev. Lett. 81, 4516 (1998).

${ }^{26}$ R. E. Camley and R. J. Astalos, J. Magn. Magn. Mater. 198-199, 402 (1999).

${ }^{27}$ R. E. Camley, B. V. McGrath, R. J. Astalos, R. L. Stamps, J.-V. Kim, and L. Wee, J. Vac. Sci. Technol. A 17, 1335 (1999).

${ }^{28}$ J.-V. Kim, R. L. Stamps, B. V. McGrath, and R. E. Camley, Phys. Rev. B 61, 8888 (2000).

${ }^{29}$ B. V. McGrath and R. E. Camley, J. Appl. Phys. 87, 6430 (2000).

${ }^{30}$ T. J. Moran and I. K. Schuller, J. Appl. Phys. 79, 5109 (1996).

${ }^{31}$ R. Jungblut, R. Coehoorn, M. T. Johnson, Ch. Sauer, P. J. van der Zaag, A. R. Ball, Th. G. S. M. Rijks, J. aan de Stegge, and A. Reinders, J. Magn. Magn. Mater. 148, 300 (1995).

${ }^{32}$ A. L. Dantas, G. O. G. Rebouças, A. S. W. T. Silva, and A. S. Carriço, J. Appl. Phys. 97, 10K105 (2005).

${ }^{33}$ A. L. Dantas, G. O. G. Rebouças, and A. S. Carriço, J. Appl. Phys. 105, 07C116 (2009). 